\title{
Civilisations
}

Revue internationale d'anthropologie et de sciences

humaines

$49 \mid 2002$

Pain, fours et foyers des temps passés

\section{Structures de combustion et préparation des végétaux de la Préhistoire récente et de la Protohistoire en France méditerranéenne}

Jean Gascó

\section{OpenEdition}

Journals

Édition électronique

URL : http://journals.openedition.org/civilisations/1810

DOI : 10.4000/civilisations. 1810

ISSN : 2032-0442

\section{Éditeur}

Institut de sociologie de l'Université Libre de Bruxelles

\section{Édition imprimée}

Date de publication : 3 juin 2002

Pagination : 285-309

ISBN : 0009-8140

ISSN : 0009-8140

\section{Référence électronique}

Jean Gascó, «Structures de combustion et préparation des végétaux de la Préhistoire récente et de la Protohistoire en France méditerranéenne », Civilisations [En ligne], 49 | 2002, mis en ligne le 01 juin 2005, consulté le 19 avril 2019. URL : http://journals.openedition.org/civilisations/1810 ; DOI : 10.4000/civilisations. 1810

Ce document a été généré automatiquement le 19 avril 2019

(c) Tous droits réservés 


\title{
Structures de combustion et préparation des végétaux de la Préhistoire récente et de la Protohistoire en France méditerranéenne
}

\author{
Jean Gascó
}

Ce texte est partiellement inspiré d'un cours donné en 1995 au Centre National d'Enseignement à Distance du Ministère de l'Education Nationale, France.

\section{Introduction}

1 Les conditions générales de conservation des sites font que l'alimentation végétale est très mal connue d'un point de vue quantitatif pour les périodes pré - et protohistoriques. On sous-estime certainement son importance. Les données archéologiques sur l'agriculture, les vestiges propres de végétaux et les dispositifs de préparation sont cependant qualitativement de mieux en mieux connus. Mais les sources sont généralement indirectes : objets (meules, faucilles), charbons surtout (champs, semences, végétaux), ou structures de préparation (poteries, foyers) etc. La présence de ces témoins indirects est la source principale des hypothèses avancées qui s'appuient sur une part évidente de bon sens. Elle peut être comparée à celle de certaines structures de combustion propres à la préparation des végétaux dont un inventaire typologique est ici proposé, ouvrant davantage de questions qu'établissant des certitudes.

2 Nous allons aborder une présentation typologique des structures liées à la préparation alimentaire des végétaux; nos exemples sont pris presque exclusivement en France méditerranéenne et pour la «civilisation de la soupe » (G. Tillion, 1966) c'est-à-dire la préhistoire récente; ils concernent principalement les structures de combustion et concernent les périodes du Néolithique et des âges des métaux. 


\section{Les structures archéologiques : quelques principes d'études}

«Parce que les faits observables n'apparaissent pas dans un ordre fixe, ni dans la fouille, ni dans l'esprit du fouilleur, il n'a pas été établi d'ordre préférentiel dans la hiérarchie des faits observés " (André Leroi-Gourhan).

Mais il convient de rappeler quelques principes d'études qui nous paraissent indispensables et qui pourront apparaître à certains comme malheureusement réducteurs (J. Gascó, 1980).

4 Nous considérons sur le terrain les faits comme primordiaux. Chaque structure est unique, individuelle. C'est l'analyse précise des formes, des composantes et des organisations des vestiges enregistrables (ou qui ne pourront l'être qu'en dehors de la fouille) qui doit être privilégiée avant le classement au titre d'un quelconque fait archéologique général (cela est valable pour les foyers comme pour les fosse, trous de poteau, murs, etc.). Nous discutons donc la notion même d'une éventuelle hiérarchie systématique de ces caractéristiques au moment de l'observation et pensons qu'une structure ne peut être réduite sur le terrain à une simple liste de données comme pour remplir une fiche type. L'approche naturaliste doit être pour nous, dans l'état actuel de nos découvertes, largement privilégiée.

5 Il ne faudrait pas cependant opposer totalement ces considérations à celle qui définit la structure ou le fait archéologique comme «une série d'unités stratigraphiques correspondant à un ensemble, structuré volontairement, qui peut-être isolé et étudié en tant que tel » (M. Py, 1991). Mais les situations sont toujours nettement plus complexes et sur le terrain les structures, (particulièrement les fosses et les foyers) ne sont pas, loin s'en faut, « évidentes » et propres à être rapidement désignées par un mot-clé.

On doit donc s'interroger sur l'affirmation qui répond aux dispositions d'esprit précédentes : « (..) il est évident qu'il existe toujours un choix, un filtre préalable, qui détermine la façon dont l'enregistrement va être effectué. Dans le cas des fouilles de Lattes (Hérault, France), ce choix est défini principalement par référence à une volonté d'interprétation historique de l'évolution du site. Ce but général suppose que les données enregistrées sur le terrain soient déjà structurées en vue d'un traitement défini. »

7 Doit-on filtrer l'enregistrement des données en fonction d'une seule problématique ou réaliser un catalogage descriptif suffisamment ouvert pour autoriser d'autres réflexions? La réalité, à Lattes, est dans la pratique souvent proche de cette seconde situation.

8 Dès lors, notre proposition ne sera pas la constitution d'un vocabulaire de mots-clés, ni celle de modèles d'installations, permettant à partir d'une identification d'envisager des types de faits ou descriptions débouchant sur des fonctions potentielles, puis probables, enfin établies.

9 A de très rares exceptions près, il est très difficile de démontrer que les dispositifs construits que nous étudions servaient effectivement à la préparation de produits végétaux. Il est évident qu'aucun foyer spécifique à ces pratiques n'est réellement connu, $\mathrm{y}$ compris les très rares et hypothétiques fours dits à pains qui permettent aussi la cuisson des viandes et des aliments en pots. Nous y reviendrons.

10 Nous rappellerons, avant tout développement, quelques considérations sur les structures évidentes; sans tomber dans l'illusion du catalogue, les grandes familles de témoins 
(Fig. 1), seront illustrées par des exemples précis. Les exemples sont pris principalement dans nos travaux ou ceux d'archéologues méridionaux; mais d'autres cas exemplaires appartiendront au domaine de la France septentrionale ou atlantique.

\section{De l'état de découverte à la forme originelle}

11 En conservant la terminologie d'A. Leroi-Gourhan, nous dirions volontiers qu'une structure est surtout « évidente » lorsqu'on le décide ! La répétitivité de la structure n'est pas un critère suffisant. Le groupement des vestiges d'origine anthropique peut toujours résulter d'une action simplement naturelle; les causes peuvent provenir du domaine minéral, animal ou végétal.

12 L'état de découverte d'une structure évidente tient en réalité à sa résistance à l'érosion naturelle : elle variera selon que la structure est homogène ou non, que son hétérogénéité ou sa granulométrie est importante, que sa composition est plus ou moins imperméable aux effets thermiques et résistante à la dissolution chimique.

13 Les effets de l'eau, du vent ou des écarts de température ont altéré les structures anthropiques depuis leur réalisation. L'archéologue admet encore plus logiquement que l'homme est également un agent qui intervient dans le degré de leur conservation; les structures peuvent ainsi être en place, aménagées, réutilisées, remaniées, dévolues à d'autres fonctions, évacuées, dispersées, décomposées, etc. C'est généralement le cas, car il est illusoire de croire que les témoins découverts témoignent directement d'un usage ultime (J. Gascó, 1980).

14 Le cas s'est posé pour l'interprétation de certains dépôts lenticulaires argileux de sites lacustres de Suisse occidentale (Auvernier-La Saunerie) dont la formation a été en son temps discutée (P. Pétrequin, 1986). S'agissait-il d'un sol de maison à pierres brûlées, avec ou non une couche d'argile de finition, ou encore de structures complexes de rejet dues à l'utilisation et à la réfection de foyers?

\section{En dehors des structures éventuelles de cuisson des végétaux, quels témoins?}

15 Existe-t-il des installations domestiques, le terme étant à prendre au sens élargi de son acception, qui ne soient pas des dispositifs liés à une action du feu ?

16 On sait que la majorité des végétaux sont consommables crûs et ne nécessitent pas, mis à part d'éventuels lieux de lavages, épluchages ou découpages, d'installations de préparation spécifiques. D’autres sont à détremper pour les laver, et surtout pour enlever de l'âcreté et les attendrir. On réalise alors un blanc, destiné à conserver ou à ôter l'amertume d'un végétal : le blanc est un mélange de farine et d'eau ou lait et d'un acide naturel (plante, vinaigre, fruit, salive).

17 Ces opérations ne donnent pas lieu à la réalisation de structures particulières, peut-être tout au plus d'hypothétiques fosses à eaux (revêtement de peaux, objets de bois enterrés, etc.) qui pourraient peut-être exister dans certains sites mésolithiques par exemple (Roc de Dourgne, Aude, France) particulièrement avant que les récipients de terre cuite n'apparaissent. Ces derniers pouvaient être utilisés hors du feu pour faire chauffer de l'eau par plongeon de pierres chauffées, accessoires fréquents des foyers (abri de Font- 
Juvénal, Roc de Dourgne). Il est probable que le développement des techniques de cuisson à l'eau tiède (blanchir : une méthode pour cuire superficiellement certains végétaux par immersion dans d'un liquide chaud, enlevant amertume et fermeté) ou de cuisson à l'eau bouillante (pocher : cuisson dans de l'eau chaude frémissante) ont pu être développées avec la généralisation de la céramique et de la consommation des céréales ou des légumineuses.

Dans certains cas, on soupçonne dans les habitats la présence de lieux et de dispositifs qui se prêteraient à la préparation des végétaux cuits ou crûs. Signalons ainsi comme exemple la recomposition d'une plaque de travail à partir de fragments d'une dalle rocheuse en grès fin, volontairement choisie et amenée sur le site de la Croix de Massargues (Saint-Martin-de-Londres, 34) ; la roche plate était brisée mais recomposée comme un mauvais puzzle pour en modifier la forme d'origine. Elle était placée, à l'extérieur d'un habitat, sous un appentis; là se côtoyaient plusieurs petits récipients, certains empilés les uns dans les autres; le sol était encombré, comme sur une aire d'habitat, mêlant ossements fragmentés, tessons de céramiques, lames et éclats de silex, broyons, charbons de bois et glands carbonisés regroupés en plusieurs petits amas (Chalcolithique, groupe de Fontbouisse, Languedoc, France).

Qu'il s'agisse de produits de la cueillette (arbouses, pistaches, olives sauvages, raisins vierges, mûres, pommes, baies diverses, glands, noisettes, cornouilles, etc.) ou de la culture (céréales, légumineuses, etc.), les données propres à la préparation des aliments et à leur consommation restent pratiquement inconnues. Et donc du domaine de l'anecdote.

On sait pourtant que les meules, broyons, etc. signalent des activités de mouture ou de concassage (concasser : écraser ou hacher sommairement). Certaines font l'objet d'une véritable installation de cuisine comme c'est le cas dans les maisons de Boussargues (Chalcolithique Fontbouisse) ou du Laouret - fig. 3-6- (Bronze final, Languedoc occidental, France). Elle témoigne de la part majeure des préparations des graines et fruits dans l'activité de la maison, ce qui se traduit spatialement par une zone d'activité nettement définie. Mais on ignore si la consommation de farines délayées, de pâtes ou de purées concassées crues ou fermentées était pratique courante.

21 Du côté des produits, il semble que cela soit attesté. Quelques pistes s'ouvrent peut-être en effet avec la découverte à la grotte des Planches (Arbois) d'un résidu d'orge concassée (interprétée il est vrai comme signalant la préparation d'une bière) et cuit accidentellement (?); on peut aussi s'interroger sur les si nombreux glands - qui sont consommables crûs -trouvés carbonisés (accidentellement?) dans de nombreux sites néolithiques ou des Ages des métaux du Sud de la France.

Par contre peu de choses nous sont parvenues pour ce qui est des exemples d'aménagement pour le séchage (et non le grillage) ou la dessiccation des végétaux (fruits, herbes) destinés à être mangé ou à constituer les produits d'allumage (friction de bois) des foyers (herbes, balles des chardons, mycelliums, etc.). Le cas d'une claie ou plaque de séchage, constituée d'un clayonnage enduit d'une argile fine lissée, découverte dans la maison du Laouret (Floure, Aude) reste bien isolé. Elle devait être suspendue au dessus du foyer installé dans la maison de bois et elle assurait surtout le rôle de pare-feu pour en protéger la toiture combustible.

D'autres dispositifs de séchage seront à aborder au titre des foyers ; ils s'apparentent aux méthodes de fumage qui dépose, par la combustion lente ou ralentie de certains bois des 
goudrons partiellement antiseptiques sur les aliments souvent traités auparavant par dessiccation ou salage.

\section{Cuissons et structures de combustion}

\subsection{Introduction}

D'un point de vue technologique, la présentation qui suit cherche à établir les correspondances entre foyers, au sens large du terme, et les préparations végétales. Elle repose sur une certaine pratique culinaire comparative et des observations sociologiques (en fait ethnographiques) répandues.

Nous n'aborderons pas une idée qui nous semble majeure, à savoir qu'au-delà de la fonction, le type de foyer reflète une culture propre, à travers ses coutumes alimentaires et culinaires.

Rappelons simplement la grande variabilité des interdits ou des obligations qui lient à des cultures complexes certaines formes de foyers et plus encore leurs pratiques. Nous donnerons simplement deux exemples volontairement matériels. J. Emperaire rapportait ainsi cette tradition alakaluf (Patagonie) : "Aucune pierre, c'est là un interdit strictement observé, ne doit délimiter la surface du feu ou servir de support aux différents ustensiles " (J. Emperaire, 1955). Dans le Rajasthan, F.Cousin observe sur un autre plan que l'orientation du foyer n'est pas neutre ni vers l'est ni vers le sud pour « ne pas sembler entrer en compétition avec le soleil, ou « ne pas ajouter de flamme au soleil ».

Pour nos périodes d'études, les indices existants sont trop peu nombreux régionalement, souvent mal définis ou mal analysés. On se reportera à titre de comparaison à la grande variabilité des structures que nous avions appelée, il y a plusieurs années, "trous de combustion » pour que le terme de « four polynésien » ne soit pas galvaudé .

Rappelons dans le même ordre d'idée que les céramiques à fond plat qui doivent avoir été perçues comme une avancée technologique à la fin du Chalcolithique en Europe occidentale témoignent indirectement de nouvelles pratiques culinaires liées à de nouveaux dispositifs de foyers. A la recherche des systèmes de calages pour des fonds ronds a succédée celle de la réalisation de surfaces planes pour placer les récipients sur le feu ou surtout à côté du feu (ce qui fait alors songer aux préparations des soupes dans nos « modernes » cheminées). Il semble que la sole de combustion de la fin de l'âge du bronze soit un des aboutissements d'une évolution des aménagements domestiques. 
Fig. 1 : Tableau des principaux types de foyer permettant la cuisson des végétaux, particulièrement des galettes, boulettes et pains, selon leur usage possible ou probable. Les utilisations autres artisanales ou domestiques sont rappelées.

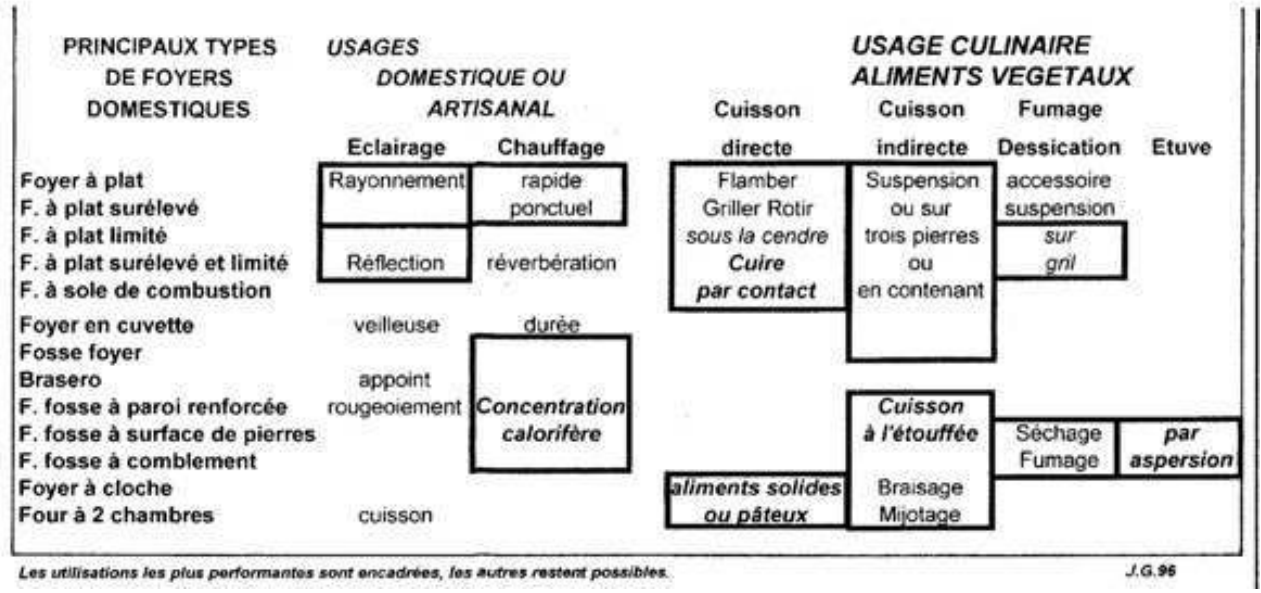

Les structures de combustion en liaison avec la préparation des végétaux peuvent être regroupées en familles généralement distinctes selon leurs composantes (charbons, cendres, pierres, terres) et leurs formes ou dispositions. Il faut toutefois préciser que les cas de relation charbons étudiés-structure étudiée sont encore exceptionnels. Nous ferons parfois allusion aux structures latentes et donnerons quelques exemples caractérisés.

Encore faut-il souligner que les structures décrites ne relèvent que d'un état de conservation et que rien ne permet d'affirmer (dans la presque totalité des cas) que nous sommes en présence d'un dispositif en état de fonctionnement ou en état d'un dernier usage conforme à son emploi commun. De même la plupart des cuissons de végétaux, y compris les préparations de galettes ou de pains levés, peut être réalisée sur toutes sortes de foyers.

1 L'ethnologie nous en apporte, s'il le faut, de nombreux témoignages: "Pour manger avec ce poisson, Naga, sa mère, fit cuire du pain dans un four circulaire en argile. Afin de travailler la pâte humide des galettes, elle la lançait avec force contre le mur intérieur de la hutte! Sur la terre ferme, il y avait un four semblable devant chaque maison. Dans les marais, les femmes cuisaient en général le pain dans des plats ronds en terre posés sur le feu » (W. Thesiger, 1959).

2 Les accessoires de cuisson ne sont pas indispensables, et si l'on connaît en Jura des plats à pain du Néolithique moyen, rares sont les groupes qui y ont fait appel. Si certaines jattes à carène très basses (néolithique moyen chasséen méridional, néolithique final vérazien) sont peut-être des ustensiles de ce type comme par exemple les coupes aplaties des VII $\mathrm{VIII}^{\mathrm{e}}$ siècles avant J.-C., il est très probable que l'on usait davantage du sol naturel, d'un galet ou d'une pierre (comme pour cuire les tortillas de l'ancien Mexique)

Les cuissons en marmite, à l'étouffée, à l'étuvée, ou encore en braisage peuvent être réalisées sur n'importe quels feux (braiser : cuisson lente et douce, avec l'apport d'un peu d'eau dans un récipient fermé; cuisson à l'étouffée: proche du braisage, sans eau, généralement sans récipient; étuver : cuire en vase clos avec la vapeur de l'aliment; mijoter : cuisson très lente, à feu doux). Les grillades sont tout autant possibles sur n'importe quels feux selon que l'on utilise ou non un gril ou une surface quelconque de chauffe (pierres, bois, etc.). 
eut se poser la question de l'existence d'une évolution technologique des foyers qui tiendrait à l'accroissement apparent de leur efficacité ou de leur commodité. C'est sans doute le cas sur la longue durée. Mais certains dispositifs différents et aux vocations particulières (feux à plats ou limités ou en fosses) ont coexisté longtemps jusqu'à ce qu'un modèle unique de foyer domestique ne s'impose ( $\mathrm{VI}^{\mathrm{e}}$ siècle peut-être) dans le cadre de l'équipement de la maison.

Il serait nécessaire aussi de maitriser la nature des combustibles utilisés, en particulier de vérifier, dater et mesurer l'usage probable des excréments d'animaux (P. Deffontaines estimait que trois vaches et sept moutons suffisaient à approvisionner une famille en combustible pour l'hiver dans les Alpes françaises) (P. Deffontaines, 1972). Le cas pourrait se poser dans certains sites où des amas de fumier brûlés ont été découverts (Abri de Font-Juvénal).

\subsection{Les structures à plat}

La cuisson des fruits, des racines, et des produits plus élaborés comme les galettes ou les pâtes végétales diverses peut être réalisées à même les braises. Plus souvent il est prudent d'attendre la constitution de cendres. On les cuit aussi en éloignant du feu, en les posant à proximité des braises, sur des surfaces naturelles (celles du feu : roche, sol durci, pierres apportées et chauffées, peaux, autres végétaux enveloppant, etc.) ; sont utilisées aussi les cuisson sur des éléments façonnés (en treillis de bois verts, sur des plaques mobiles de cuisson comme les possibles plats à pain chasséens (Fig. 2) du Nord de la France, à l'intérieur de vase ouvert, sur la panse retournée de grande céramique, etc.). Dans tous ces cas, le foyer à plat reste parfaitement adapté.

Mais, de la possibilité à la réalité du fait, il semble impossible de trancher. Nous nous contenterons dans un premier temps de présenter les structures en développant quelques arguments pour affiner cette première approche.

Fig. 2 : Céramique du néolithique moyen du Jura, Le Camp de la Roche (Besançon, Doubs) dite plat à pain $(14,5 \mathrm{~cm}$ de diamètre $)$

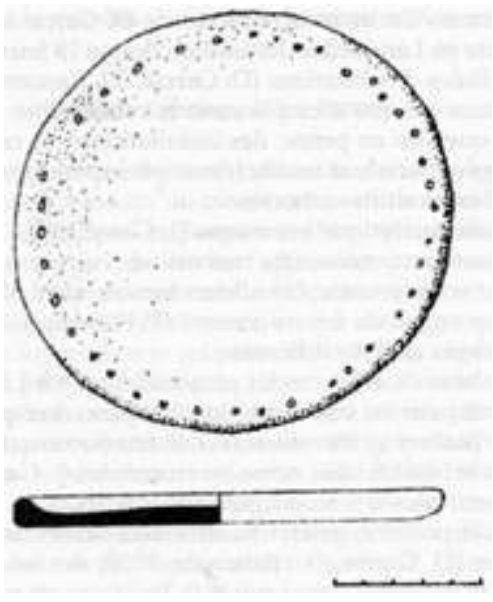

\subsubsection{Les aires de foyers successifs}

Nous signalerons d'abord ces installations pour mémoire. Les aires de foyers successifs et partiellement superposés sont fréquentes dans les abris sous-roches ou en grottes lorsque 
des données physiques de position par rapport à un courant d'air ou une protection de dalle favorisent certains lieux pour allumer un feu. Ces aires sont parmi les plus difficiles à fouiller pour mettre en évidence les relations entre les foyers et leur contextes archéologiques (abri de Font-Juvénal). On doit les considérer comme des feux simples. Ces aires livrent fréquemment de nombreux macro-restes végétaux (graines) lorsque les combustions ne sont pas abouties. Des usages artisanaux (grillages des céréales pour dépiquetage ou conservation, etc. ) sont sans doute à envisager.

\subsubsection{Les foyers à plat simples}

39 Ces foyers sont les simples traces des feux allumés sur le sol. Ce ne sont pas des marqueurs chronologiques sur un plan typologique. Ils représentent à toutes époques au minimum un feu sur deux étudiés. Encore faudrait-il préciser que les feux étudiés sont rares. Un inventaire récent de D. Garcia et G. Rancoule pour la Protohistoire récente en Languedoc-Roussillon donne 18 feux connus, sur cinq sites et cela pour 392 cellules d'habitations (D. Garcia, G. Rancoule, 1989) ! Et encore, il ne s'agit dans certains cas que d'emplacements rubéfiés.

Ce sont, quoi que l'on en pense, des installations très caractéristiques, mais le plus souvent négligées dans leur fouille (description, stratigraphie, contenus) à l'exception peut-être des produits carbonés.

41 Leur vocabulaire analytique est connu (J. Gascó,1980, 1985, A. Beeching et J. Gascó, 1989) mais souvent encore très mal utilisé (voir par exemple les notions de dépôts lenticulaire ou semi-lenticulaire, leurs formes, etc.). Nous les distinguons de la terminologie trop vague de foyers ouverts (P. Pétrequin, 1986, p. 296, qui décrit sous ce terme plusieurs cas très différents).

Leur taille avoisine dans les cas les plus anciens 0,8 à $1,2 \mathrm{~m}$ de diamètre. Cette dimension fréquente pour les structures domestiques correspond à une ergonomie, une pratique pour réaliser et travailler avec le feu (artisanat, et surtout cuisine ou chauffage). Durant le Néolithique, nous avons montré (J. Gascó, 1980) que souvent plus le foyer est petit, plus ses aménagements intérieurs ou extérieurs sont nombreux (petit calage de potence, galets chauffés accessoires, tripode de roches, etc.). Durant l'âge du fer (D.Garcia, G. Rancoule, 1989) ces feux pourraient être plus petits $(25 \mathrm{à} 50 \mathrm{~cm}$ de diamètre de sol rubéfié). Ils évoquent pour nous des feux d'éclairage ou de petits dispositifs d'appoint, à moins qu'ils ne signalent des usages spécifiques individuels (particulièrement dans les espaces ouverts).

Les foyers à plats permettent la cuisson sous la cendre des galettes ou boulettes, à la manière des «batis » indiens (F. Cousin, 1989) ou des « rondelles de pains bis brûlées par endroit et maculées de cendres » du Proche Orient (W. Thesiger, 1959). C'est même sans doute le feu le plus employé à cet usage, tant que les ajouts de ferments n'ont pas été l'usage pour préparer les pains qui alors nécessitèrent des cuissons aériennes ou en four. Il existe cependant une technique consistant à cuire les aliments (sans liquide) dans une céramique fermée et dont on chauffe les parois à la manière d'un petit four.

Certains foyers ont été utilisés de nombreuses fois et leur nettoyage (vidange, raclage) ont pu en surcreuser le soubassement. On peut parfois observer à proximité des structures d'évacuation ou de réutilisation des braises. Les foyers sont repérées par leurs cendres recouvrant parfois des charbons de bois, ou également auréolant les amas. La base des installations porte des marques de chauffe : rougissement des minéraux, parfois éclats 
thermiques, etc. Dans certains cas, le soubassement du foyer, constitué d'éclats calcaires, peut être calciné et la chaux qui se forme à la surface des pierres se transforme en un véritable mortier induré, qui se colore parfois de rouge au contact d'oxydes argileux du sédiment; c'est le cas dans les cavités karstiques à éboulis secs (Grotte de la Garenne, Penne, Tarn). Il existe des cas où des accessoires de cuisson (gril constitué de pierres, tripodes pour la stabilité des marmites, calage de piquet) accompagnent ces installations (J. Gascó, 1980, P. Pétrequin, 1986). Le cas des foyers chasséens couverts de pierres est cependant à distinguer car ils appartiennent à des structures en creux. L'étude précise des stratigraphies des foyers à plat peut conduire à des éléments d'interprétation fonctionnelle (Abri du Roc de Dourgne, Aude, France).

En plein air, seuls les plus grands foyers à plat paraissent être conservés sur les grands sites; ceci pourrait exclure leurs utilisations pour les cuissons de végétaux, bien que le caractère collectif de certaines pratiques ne puissent pas être exclu (c'est d'ailleurs une hypothèse avancée pour certains foyers du Néolithique moyen chasséen). Mais il est possible que des problèmes de conservation pour de plus petites installations se posent.

\subsubsection{Les feux à plats aménagés sur des plates-formes}

Il semble qu'il s'agisse d'une variante construite du foyer à plat. Il s'agit d'isoler le feu du sol combustible lorsqu'un plancher constitue l'assise de la maison. Le cas du foyer $n^{\circ} 2$ de Charavines-Les Baigneurs (Néolithique final) a été très bien décrit. L'aire du foyer a été entretenue avec l'adjonction progressive de chapes d'argile au fur et à mesure de l'enfoncement de la structure dans la craie lacustre du village. La stratigraphie qui en résulte montre les aménagements multiples que devaient subir tous les foyers. Sur le sol couvert de débris végétaux, une première chape d'argile couvrait près de $2 \mathrm{~m}^{2}$. Après l'incendie et l'abandon du site, le dôme qui subsistait a été réutilisé, près de 30 ans plus tard (dendrochronologie). En plusieurs phases de construction, on installe une sole d'argile à cailloutis sur des planches de récupération ou de petits troncs refendus ; elle est limitée en dernier lieu par une planche verticale maintenue par deux piquets. De petites bordures d'argile existent parfois. De nombreux foyers de ce type ont été fouillés à Charavines, à Clairvaux-les-Lacs, et dans de nombreux sites palustres (P. Pétrequin, 1986).

De tels aménagements semblent se limiter pour l'instant au domaine lacustre. Mais ils répondent à des impératifs de sécurité dans des constructions en bois; ils montrent une volonté de limiter et localiser le feu, le souci d'effectuer des chauffes limitées (ce qui convient parfaitement à la cuisson des galettes), avec des branches partiellement consumées et créant des rubéfactions très superficielles de leurs argiles qui pouvaient cependant réverbérer une certaine chaleur. Dès lors, il est possible que de telles installations puissent être découvertes dans des maisons davantage «terrestres " du Néolithique. Il faut sans doute rattacher à cet ensemble les foyers sur radiers de blocs (Font-Juvénal en Languedoc, France ; Cougevaux-en-Triva, Fribourg, Suisse). L'ethnologie pourrait les rapprocher des «fucone ou ziglia » corses du XVIII ${ }^{e}$ siècle (P. Arrighi, 1970, p. 41).

Ce type de foyer est une variante du feu à plat dont les preuves d'un choix raisonné constructif sont évidentes, mais très peu éloignées de celles généralement réunies dans les cas ordinaires: emplacement, vent, horizontalité, nature du sol, nature de la couverture, questions de limite, de la durée, du combustible, de son évacuation, etc. Il est 
donc nécessaire de les distinguer des soles de cuissons bâties qui apparaissent au cours de l'âge du Bronze ou du Fer dans le Midi de la France.

\subsubsection{Les soles bâties de cuisson}

49 Ce sont des foyers matérialisés par une sole de cuisson formant une chape non bordée. La construction mêle le plus souvent à l'argile, sélectionnée pour sa plasticité, et provenant de cavités parfois éloignées du site, des pierres parfois, ou encore des tessons de céramiques choisis pour leur platitude qui participent à un soubassement. En milieu palustre des aménagements proches sont constitués aussi de dalettes ou encore de morceaux d'écorces (Egolzwil) (P. Pétrequin, 1986). On signale des coquillages dans certains cas en Provence protohistorique (N. Nin, 1989).

Il ne s'agit pas d'isoler le feu du sol de la maison mais bien d'en marquer la limite précise et surtout de réaliser un accessoire particulier propre à certaines cuissons. Il est clair qu'un tel dispositif, et c'est peut-être son usage principal au cours de l'âge du fer, peut être utilisé comme simple emplacement de foyer à plat, bien délimité dans les habitations. Mais il nous semble pouvoir être mis souvent en relation directe avec une technique particulière de cuisson des pains et galettes fines.

Il est parfois difficile de distinguer typologiquement de telles installations sur sol naturel de certains foyers construits sur planchers en bois. Quelques descriptions réunies pour les maisons des tourbières de Bavière par P. Pétrequin l'indiquent bien (P. Pétrequin, 1986). Il semble que certains dispositifs des milieux palustres appartiennent aussi à cette catégorie (Riedschachen, Federseemoor, par exemple), la distinction se faisant peut-être à travers l'utilisation ou non de dalettes calcaires susceptibles de conserver la chaleur du foyer.

Ces foyers sont considérés par certains protohistoriens comme "construits", terme impropre au regard de l'organisation des autres foyers tout autant élaborés.

Durant la Protohistoire récente, lorsqu'ils deviennent le foyer type de l'habitation de l'âge du fer méridional, $41 \%$ sont constitués d'argile seule déposée en chape lissée. $30 \%$ seraient placés sur un lit de blocailles, $9 \%$ sur des tessons, $7 \%$ sur des tessons et des pierres (D. Garcia et G. Rancoule, 1989).

Ces dispositifs ont des formes rondes pour les plus anciens, parfois carrées (particulièrement à l'âge du fer, dans $65 \%$ des cas étudiés en 1989); les plus récents à l'âge du fer ont parfois leurs bordures décorées de cannelures, (dix cas connus en 1989 datés des VI-IV ${ }^{e}$ siècles en Languedoc oriental), parfois de motifs géométriques estampés (grecques) et sont accompagnés de chenets mobiles en terre cuite (foyer de la maison I de Gailhan, Gard, milieu de l'âge du fer). Leur préparation est soignée.

Mais ces aménagements sont fragiles et, à la fouille, on les trouvent souvent démantelés. Leur surface est alors simplement estimée (de 0,6 à $1 \mathrm{~m}^{3}$ à Quitteur, bronze final IIb, Haute-Saône). Sur certains sites, il ne s'agit que d'une chape d'argile ovalaire flanquée sur quelques petits blocs calcaires placés à même le sol (en Lorraine généralement 0,70 sur $0,50 \mathrm{~m}$ selon V. Blouet, communication personnelle).

Les étapes de fabrication pour les soles de cuisson les plus élaborées d'entre elles (nous ferons référence à un exemple du Laouret, commune de Floure, Aude, Bronze final IIIa, $\mathrm{F}$ ig. 3 à Fig. 6) montrent l'organisation de leur soubassement, parfois en cuvette, puis le dépôt d'une chape de tessons qui peut présenter plusieurs épaisseurs et/ou de celle de boules de torchis (argile et végétaux, peut-être fumier animal) qui sont alors parfois 
installées entre des interstices de clayonnage en branches, enfin le placage d'argile fine et son lissage jusqu'à obtenir un enduit plus régulier. La sole devait être décorée par une double cannelure peut-être périphérique.

Fig. 3 : Etat de découverte de la sole de combustion d'une maison au Laouret (Floure, Aude), âge du bronze final (IIla)

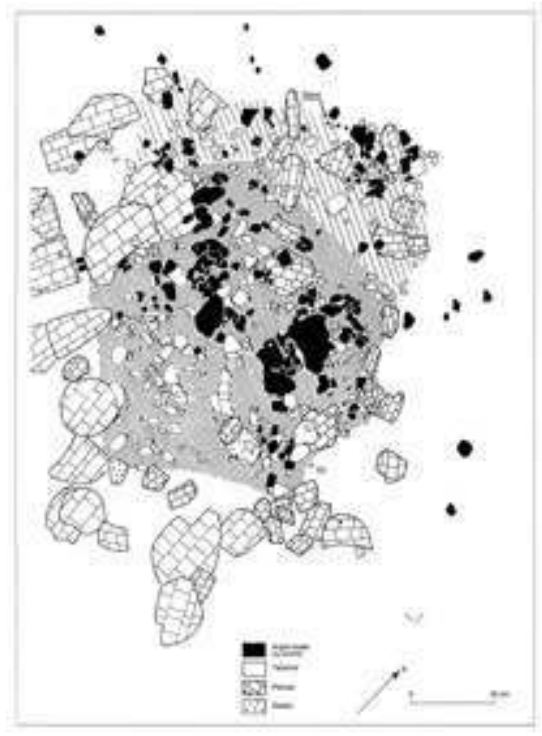

Fig. 4 : Empreintes de branchâges sur les faces inférieures des blocs de torchis de la sole de combustion d'une maison au Laouret (Floure, Aude), âge du bronze final (Illa). Les orientations des bois placés sur les bords indiquent une construction soignée ; au centre, le renforcement de l'installation, dans le sens du plus grand usage de la sole (du fait de sa position dans la maison) est à noter.

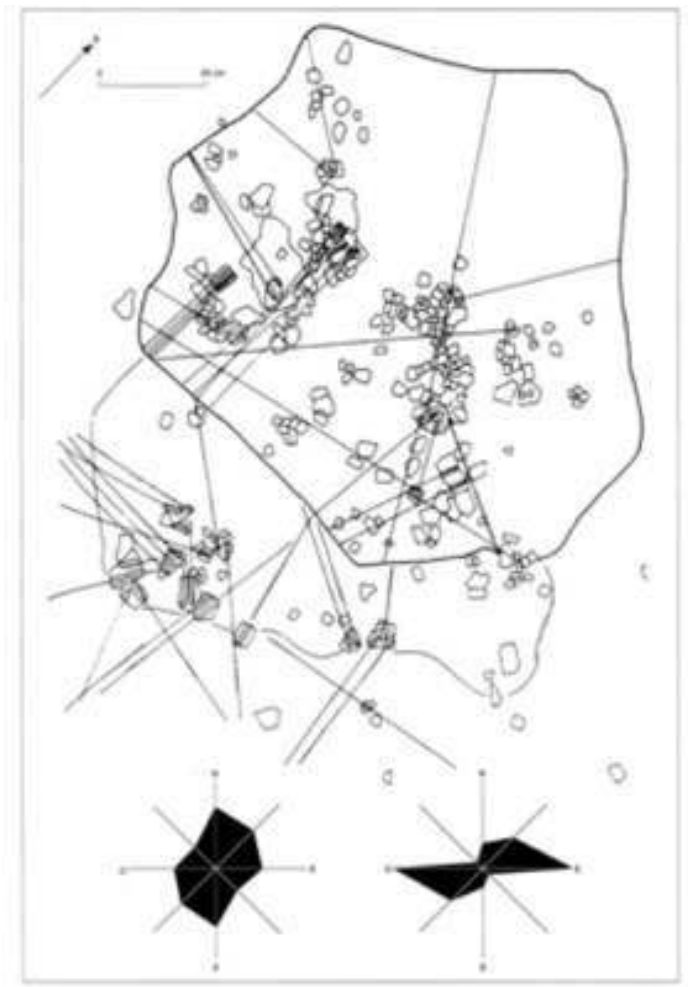


Fig. 5 : Ensemble des éléments rocheux de la sole de combustion d'une maison au Laouret (Floure, Aude), âge du bronze final (Illa) ; les alignements des blocailles résultent peut-être de mouvements dûs à l'étalement des apports d'argile lors du lissage de la sole.

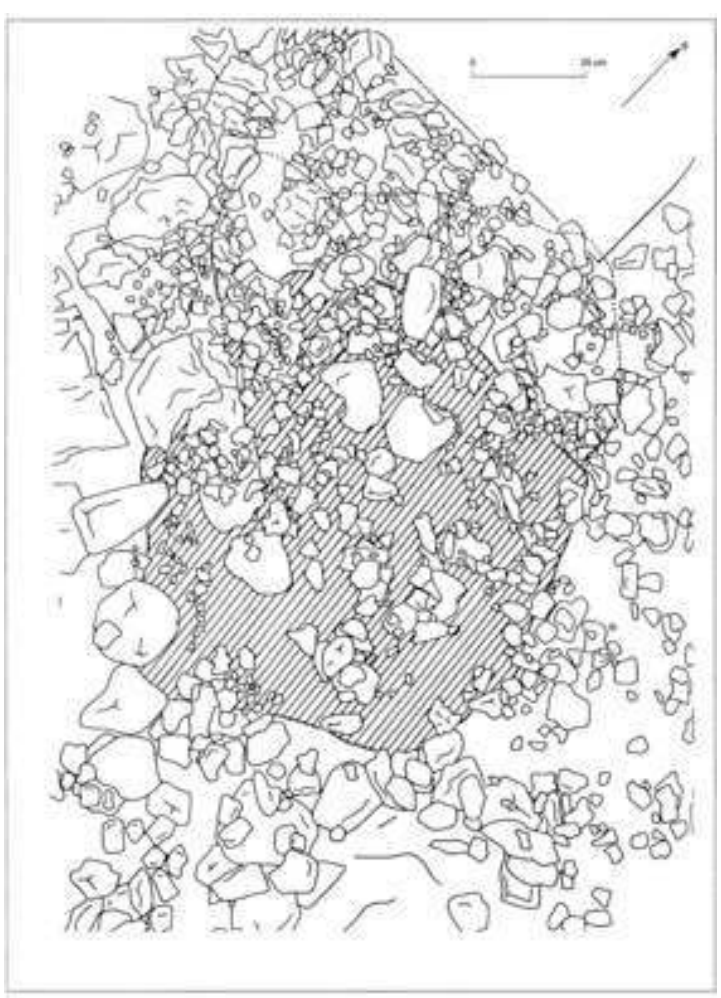

Fig. 6 : Coupe de la sole de combustion d'une maison au Laouret (Floure, Aude), âge du bronze final (IIla). Echelle: voir Fig. 3.

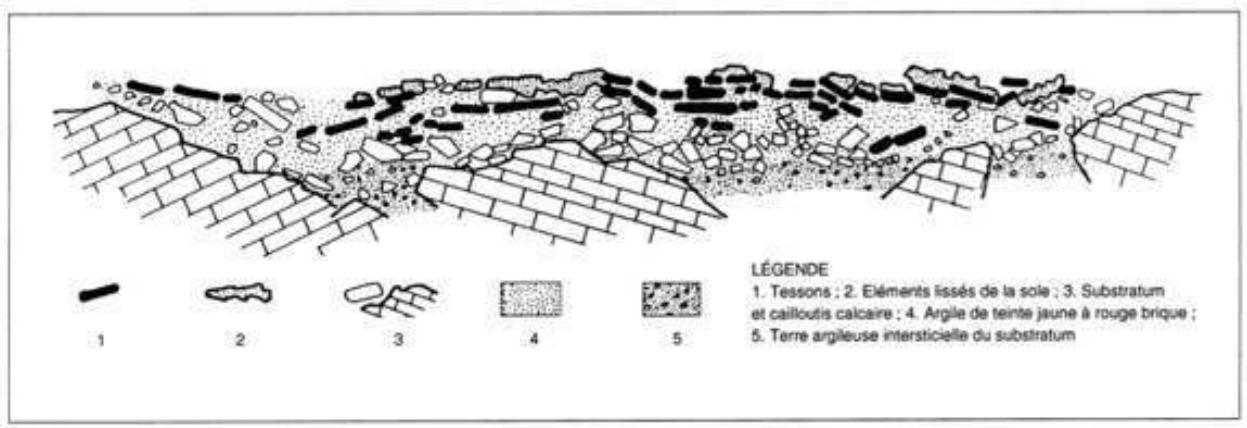

Cette construction précise n'évite pas de fréquentes réparations; elles étaient réalisées par adjonction d'argile, ou parfois (comme à l'abri de Font-Juvénal pour un horizon tardif) par installation de lits successifs de tessons et d'argile.

Le fonctionnement de ces plaques de cuissons lie parfois deux structures de combustion : l'une, à plat ou en fosse (Le Cros, Caunes Minervois pour le VI siècle av. J. -C, Arles au Ve) permet d'obtenir des braises qui sont placées ensuite sur l'autre, la plaque de chauffe : il s'agit d'éviter un feu trop violent (flamme) à l'intérieur de constructions en bois (toiture comprise).

Dans d'autres cas, la plaque de chauffe est utilisée d'abord pour la réalisation d'un feu qui propage sa chaleur jusqu'au coeur des tessons et des argiles mêlés, puis les braises ou les 
cendres chaudes sont évacuées et la plaque à « induction » peut être à son tour utilisée. La chaleur diffusée par les brandons jusqu'à l'argile est suffisante pour que cette dernière permette la cuisson de galettes fines. On ne peut exclure l'utilisation de vase retourné pour maintenir la chaleur du foyer. Le dépôt des carbones d'enfumage est alors important.

Durant l'âge du fer (D. Garcia, G. Rancoule 1989), on peut noter que l'absence fréquente d'une réelle semelle de pierres ou de tessons ( $16 \%$ des cas) indique que son utilité thermique n'est plus recherchée. Ces structures de combustion semblent n'apparaître qu'à la fin de l'âge du Bronze en France méditerranéenne, quelque temps après leur utilisation en Europe continentale et rhénane.

\subsection{Les foyers à plat limités}

61 Il s'agit de foyers volontairement limités par un agencement de pierres (tardivement on connaît des installations limitées par des briques maçonnées comme à Entremont), parfois une murette afin de les isoler de leur environnement. Ce fait est important et doit les distinguer nettement des feux à plat. Chronologiquement, il est possible que leur nombre aille en s'accroissant à partir du Néolithique final ou un peu auparavant (FontJuvénal) jusqu'à la fin de l'âge du bronze ; mais le manque de séries de foyers étudiés peut aussi laisser à penser que l'on n'enregistre là que les modifications du statut des sites et de leur environnement (augmentation des temps de séjours, individualisation des lieux de vie, etc.). Leur dimension est comprise entre 0,6 et 1,6 m. On ne réunit pas à ce groupe les feux à plat adossés à un bloc rocheux naturel ou une paroi, qui sont à considérer dans le groupe précédent. Leur degré d'élaboration est plus faible.

62 Mais dans les deux cas, la pierre chauffée emmagasine la chaleur du feu et la réverbère lentement. Il n'est pas rare que de tels feux soient installés sur des dalles rocheuses, dans le même but. Ces foyers sont tout particulièrement adaptés à la cuisson des boulettes ou des galettes car ils permettent d'économiser le combustible; l'accumulation de la température dans les roches de limitation, avec souvent des faces planes propices au dépôt d'aliments, est leur principale qualité avec celle d'une diffusion continue de la chaleur et d'un faible abaissement de la température.

Cette sorte de chaleur est très adaptée à la cuisson des pâtes : concentration de la chaleur, refoulement de l'humidité vers le centre de la galette, création d'une coûte qui retient l'humidité résiduelle, assouplissant la pâte lors de son éloignement du foyer.

Des dallages ou des tripodes de pierres devaient recevoir les vases de cuisine, mais les pierres de chauffe y sont rares (elles seraient peut-être plus difficiles à extraire des braises à cause des blocs de limite). Certaines pierres plates ont servir de plaque de chauffe pour des galettes (Roc de Dourgne, Abri Jean Cros dans les Corbières en Languedoc).

Les limites du feu sont fréquemment interrompues par une ouverture qui permettait de nettoyer le foyer (Baume de Ronze, en Ardèche, Font-Juvénal dans l'Aude, etc.). On trouve là une indication solide pour " orienter » le foyer dans un espace domestique et trouver l'emplacement de son dernier utilisateur ou du plus fréquent (Abri du Roc de Dourgne).

Des foyers à plat limités d'anneaux d'argile modelée ont été découverts en plusieurs exemplaires en territoire de la culture de Véraza du Néolithique moyen du bassin de l'Aude. Certains pourraient cependant dater de l'âge du fer bien qu'à cette époque les 
tores qui pouvaient servir de support durant la cuisson, voire de mandrin lors de la construction des fours, soient souvent de plus petite taille (Carsac, commune de Carcassonne, Aude, Fig. 7). L'usage culinaire de tels foyers est peu probable pour les cuissons de végétaux.

\subsection{Les foyers en creux ou trous de combustion}

Nous avons longtemps proposé d'utiliser pour ces structures le terme générique de trou de combustion; au-delà de l'aspect provocateur de cette terminologie, nous souhaitions éviter les usages du terme de four: fours polynésiens ou fours canaques, ou fours souterrains ou encore fours de terre : ces derniers sont en fosse, les autres sont en amas (four canaque) proches des foyers à pierres chauffantes (connus au Canada chez les Assiboines, ou encore en Océanie).

Les formes en creux qui nous intéressent ici ne sont pas limitées à la période du Néolithique moyen où leur nombre est particulièrement important. Il en existe avant et il en existe également après. Ces foyers sont souvent associés à des feux à plat ou des épandages de cendres durant le Chasséen.

Ces structures regroupent de nombreuses variantes de foyers. Elles ont en commun leur nature, une dépression plus ou moins marquée et leur remplissage en produits de combustion. Selon leurs formes et leurs tailles, selon la composition de leur remplissage, de charbons et/ou de cendres, selon la disposition stratigraphique de ces produits, selon la présence ou non de blocs rocheux, et leur position, ces foyers en creux ou trous de combustion (terme encore plus général) appartiennent à divers types.

70 Il est clair que ces formes ne sont pas exemptes de nombreuses modifications, dues à des usages variés ou à des conservations particulières, qui doivent être examinées avec soin. Il reste aussi que dans de nombreux cas le choix de la fosse est guidé non par l'usage que l'on compte faire du foyer mais par l'état de son environnement direct (sol natté, tapis, pailles, etc.)

71 Les foyers en creux peu profonds sont souvent emplis de cendres et témoignent de combustions abouties. Ils sont proches des feux à plat. Certains foyers sont dallés de pierres. Ce sont sans doute les plus propices à la cuisson de végétaux préparés sous forme de pâte. D'autres possèdent des parois en dalles rocheuses formant caisson (FontJuvénal). Ils sont souvent appelés fours. Mais il est rare que leur état de conservation puisse en préciser exactement la forme originelle.

On doit les distinguer des foyers-fosses à remplissage de pierres. De telles structures peuvent présenter selon le moment de leur abandon des organisations différentes de vestiges : à l'abri de Font-Juvénal nous avons montré en d'autres occasions les différentes étapes des foyers-fosses qui pouvaient livrer des vestiges apparemment différents.

Ces foyers à remplissage de blocs ne paraissent pas très propices à des cuissons de végétaux à l'étouffée; toutefois le recours à l'emballage des pâtes de céréales dans des feuilles (châtaignier, mais aussi salades sauvages par exemple) s'avère un exemple d'usage possible selon cette technique, qui reste surtout efficace pour les viandes; il est vrai que de telles sources de chaleur permettent, en surface des blocs, de nombreux usages (y compris le maintien à chaleur de récipient) ; et les cuissons de galettes, ou les diverses grillades de fruits (marrons, glands sur la branche, pommes) pouvaient y être appliquées comme sur les feux à plat. 
74 Les dimensions de ces foyers paraissent être grosso modo en correspondance avec leurs organisations internes et ont permis d'affiner une esquisse typologique qui suit.

\subsubsection{Les foyers-fosses domestiques}

- Il existe de rares petits foyers-fosses approchant $0,6 \mathrm{~m}$ de diamètre. Ce sont généralement des structures complémentaires proches d'un foyer principal et à la dimension d'un usage individuel ou ponctuel. On peut y placer un récipient à fond rond. Leur utilisation pour la cuisson des galettes est possible ; on notera qu'en Inde contemporaine le dispositif le plus fréquent pour une cuisson sous cendres est celui -ci (F. Cousin, 1989).

- On le distinguera des petits braseros fixes au diamètre approchant 0,2 à $0,3 \mathrm{~m}$ seulement : ces trous sont généralement emplis de cendres résiduelles, pratiquement sans charbons et leurs parois régulières ne portent pas de traces évidentes de chauffe. Il faut rester cependant prudent si une telle structure est découverte isolée, la modification du sédiment sous l'effet du feu étant très variable d'un site à un autre ; l'ethnologie nous montre des exemples comparables de petites structures à chauffer les pierres dans les anciennes cases canaques.

- Des foyers-fosses nombreux approchent 0,8 à $1,3 \mathrm{~m}$ de diamètre ; ces dimensions les rendent comparables aux foyers à plat. Les dallages de fond sont connus (Abri de Font-Juvénal, Aude). Leur usage impose souvent des circulations autour du feu et une organisation de la zone de vie pour son approvisionnement en bois et son usage.

\subsubsection{Les foyers-fosses à blocs de pierres et les structures assimilées}

On les compare souvent aux foyers polynésiens. Leurs points communs sont la présence de pierres chauffées empilées qui forment soit des niveaux distincts (lits) soit un amas inorganisé. Les variantes livrent des preuves de chauffe poussée et achevée (remplissages de cendres) ou des indices de combustion interrompue ou partielle (brandons étouffés, suies déposées par les fumées, cendres encroûtées par un enrichissement en carbonates de chaux). Ces états de conservation traduisent les étapes de leur fonctionnement général et de leur probable utilisation.

Il est cependant possible de distinguer :

- des foyers-fosses de grande dimension au diamètre dépassant 1,5 $\mathrm{m}$ et jusqu'à 2,2 $\mathrm{m}$. Ces structures sont découvertes en plein air comme en grotte ou sous abri. Ce sont les premiers fours à chambre de chauffe enterrée qui autorisaient presque toute les cuissons par enfouissement dans des braises ou cendres chaudes, par présentation à la flamme avec rôtissage ou grillade (formes de cuissons toujours possibles) et surtout par recouvrement et cuisson à l'étouffée ou à la vapeur dans des empilements de pierres chauffées. Les viandes paraissent les plus aptes à ces types de cuisson mais les expériences que nous avons réalisé indiquent leur extension aux végétaux, surtout aux fruits. Le creusement du foyer assure une concentration de la chaleur, une meilleure et rapide combustion des bois et une forte réverbération de la chaleur qui était amplifiée par celle des roches chauffées. A Font Juvénal on y cuisait de grands quartiers de boeuf, durant la période chasséenne. On en connaît en réalité à toutes les périodes jusqu'à la fin de l'âge du bronze (par exemple Soyons-la Brégoule en Ardèche, Montagnieu le Pré de la Cour dans l'Ain etc.).

- des structures de combustion de très grande taille, trouvées en batteries sur plusieurs grands sites chasséens du bassin moyen de la Garonne (220 sur $3000 \mathrm{~m}^{2}$ à VilleneuveTolosane) dont l'organisation collective pose encore des problèmes d'interprétation. Certaines fosses ont des diamètres oscillant entre 0,8 et 2,3 m ; d'autres rectangulaires 
mesurent de 1,85 m à 11,3 m (plus de 6 fois plus) pour des largeurs constantes de l'ordre de 1,3 à $2 \mathrm{~m}$. Ces structures ont été réalisées en creusant une fosse où l'on allumait un feu de bûches ; des galets rassemblés au centre des fosses rectangulaires ou disposés en couronne dans les trous circulaires étaient chauffés. Ils étaient ensuite étalés sur toute la surface de la fosse. Leur usage est inconnu mais des hypothèses ont été avancées sur la base de structures contemporaines comparables (foyers-fosses des habitats) ou de comparaisons ethnographiques.

77 Il est possible d'envisager l'hypothèse d'une cuisson à l'étouffée ou à la vapeur (en introduisant de l'eau à travers l'ouverture d'une fermeture provisoire, avec une peau par exemple) qui expliquerait le démantèlement fréquent des stratigraphies des structures. Mais le fait d'installer des viandes à rôtir sur un gril de pierres (comme dans les foyersfosses des habitats de Font-Juvénal) ou de préférer chauffer lentement des morceaux en les recouvrant de blocs est d'ordre culinaire et non pas domestique.

En tout état de cause les multiples foyers auraient produit des quantités considérables de nourriture prête à être consommée. D'autres hypothèses vont dans le même sens : celles du fumage ou du séchage des viandes ou poissons attribuent à la sole de galet un rôle d'isolant chaud. Les structures auraient pu aussi être utilisées pour griller des récoltes de céréales pour en assurer une meilleure conservation.

79 L'idée enfin du calorifère arrosé d'eau à la manière des Amérindiens, des peuples nordiques ou des Scythes durant l'Antiquité, ouvre le champ des grandes manifestations collectives qui nous échappent encore davantage.

D'autres aménagements ronds distincts des structures rectangulaires ne doivent pas être assimilés au même modèle, comme leur distribution sur les sites (anarchiques pour les rondes, collectives pour les grandes) semble l'indiquer. Les foyers-fosses circulaires paraissent en effet pouvoir être raccrochées aux modèles domestiques connus depuis le Mésolithique montclusien et jusqu'au Chalcolithique, mais très fréquents au cours du Néolithique moyen chasséen dans le Sud de la France.

\subsection{Les foyers couverts}

81 Les foyers couverts sont dénommés fours lorsqu'ils sont maçonnés ordinairement en forme de voûte. Ces fours en terre sont connus en Europe centrale néolithique. En Europe tempérée, les éventuelles constructions sont rares et très mal conservées. L'apparition du four y reste en fait mal datée.

On en signale dans le chasséen de Saint-Michel-du-Touch ou de Villeneuve-Tolosane, mais les descriptions font défaut et dans la plupart des cas il s'agit d'indices secondaires (Vaquer 1990). Dans la plupart des cas, ce sont des fragments de plaques, de sole ou de parois de four en argile ou en torchis qui témoignent de ces installations culinaires ou artisanales probablement placées le plus souvent dans les habitations. Ces fours ne posséderaient qu'une salle de chauffe et de cuisson (au four tiède pour les pâtes qui doivent gonfler, chaud pour les grillades et toutes les cuisson en marmite).On ne peut écarter l'idée qu'il puisse s'agir de simples cloches à cuire ou de couvercles de récipients en terre levée (terre argileuse utilisée en pisé ou torchis).

83 Il est difficile d'affirmer l'usage précoce des fours construits à l'aide de clayonnages enduits d'argile; on admet en effet que la cuisson de la céramique est pratiquée à la meule, ou en fosse jusqu'à une période avancée du Néolithique. C'est pour cette raison 
que les fours soupçonnés sont attribués plutôt aux cuissons de pâtons et galettes pour l'alimentation. Rappelons que les viandes ou les poissons peuvent y trouver leur place.

Ainsi des plaques d'argile avec des empreintes de branchages (Les Valentines, commune de Saint-Jean-de-Cas, Aude) ou des entrelacs de joncs (Cassenac, commune d'Ouveillan, Aude) ont été interprètes comme des restes de fours à galettes. Certaines fosses à parois de pierres ont pu être considérées comme de petites salles de chauffe (Néolithique final, Abri de Font-Juvénal, commune de Conques, Aude). D'autres fosses sont à paroi chauffée et durcie par le feu ; nous avons repoussé la terminologie de four pour ces dispositifs qui en sont peut-être toutefois des exemplaires primitifs, proches des foyers-fosses.

On connait un cas particulier à Notre-Dame-de-Marceille (Limoux, Aude) qui est un fosse couverte; elle date du Néolithique final (groupe de Véraza vers 3500-3000 av. notre ère). C'est une fosse oblongue aux parois latérales et à l'extrémité creusée en sape présentant des traces de cuisson (durcissement, rubéfaction). A l'origine la fosse était voûtée en terre levée et il n'en subsiste que la chambre de chauffe comblée d'au moins quatre niveaux de feux. La structure est peut-être destinée à cuire la céramique, comme le pense J. Vaquer.

Les premiers fours que l'on connaisse avec quelques précisions datent de la fin de l'âge du bronze et du Premier Age du Fer. Il en existera de nombreuses variantes qui font appel à des techniques et des solutions architecturales différentes. C'est sans doute là la preuve de l'ancienneté de la construction des fours en terre. L'un d'entre eux possédait une sole de terre cuite et une coupole de torchis avec une cheminée de terre cuite (Bronze final IIIb, Les Glandus, Saint-Férréol Trente-Pas, Ardèche) (J. C. Daumas, R. Laudet, 1992). Parmi eux les fours à pains ou galettes sont encore très rares.

87 A Carsac (fin VII siècle, Carcassonne) a été découvert l'un des plus anciens d'entre eux, comparable selon J. Vaquer, aux fours de l'Agora d'Athènes (VI ${ }^{e}$ A.C.). Sur le site, plusieurs fours sont construits en pisé et en fin torchis sur une armature de branches de saule, de piquets de bois et de roseaux entrelacés; d'autres sont creusés dans la terre argileuse ( J. Guilaine, 1986).

88 Mais la fosse 7 (Fig. 7) a livré des fragments de voûte présentant une ouverture sommitale de 40 à $45 \mathrm{~cm}$ de diamètre. La base de ce four devait être échancrée par une ouverture hémicylindrique de $10 \mathrm{~cm}$ environ de rayon. Une sole probable, épaisse de 5 à $6 \mathrm{~cm}$, était très cuite avec un enduit argileux. Les parois internes du four étaient rainurées de tracés digitaux disposés en chevrons. Le couvercle amovible du four était réalisé en fin torchis lissé sur les deux faces; il porte un anneau permettant de déplacer la calotte hémisphérique avec une tige lorsqu'elle était chaude. Le rebord chanfreiné du couvercle est décoré d'une cannelure décrivant des ondulations (diamètre 50 à $56 \mathrm{~cm}$, hauteur $12 \mathrm{~cm}$ sans l'anneau d'environ $14 \mathrm{~cm}$ d'épaisseur).

89 Ce four est décrit comme un four à pain du fait de ses parois internes striées, mais d'autres usages ne sont pas écartés par J.Vaquer (grillage des céréales). D'autres dispositifs ont été découverts sur le même site : four de potier (à voûte, alandier et sole de cuisson perforée) et four de métallurgiste (avec une paroi perforée pour adapter des tuyères de soufflets). 
Fig. 7 : Couvercles de four ou cloche à cuire du site de Carsac (Carcassone, Aude) provenant des fosses 7,30 et 26 et hypothèse de reconstitution d'un four à pain (fosse 7) à partir des éléments découverts; premier âge du fer. (VIIe siècle avant J.-C.)

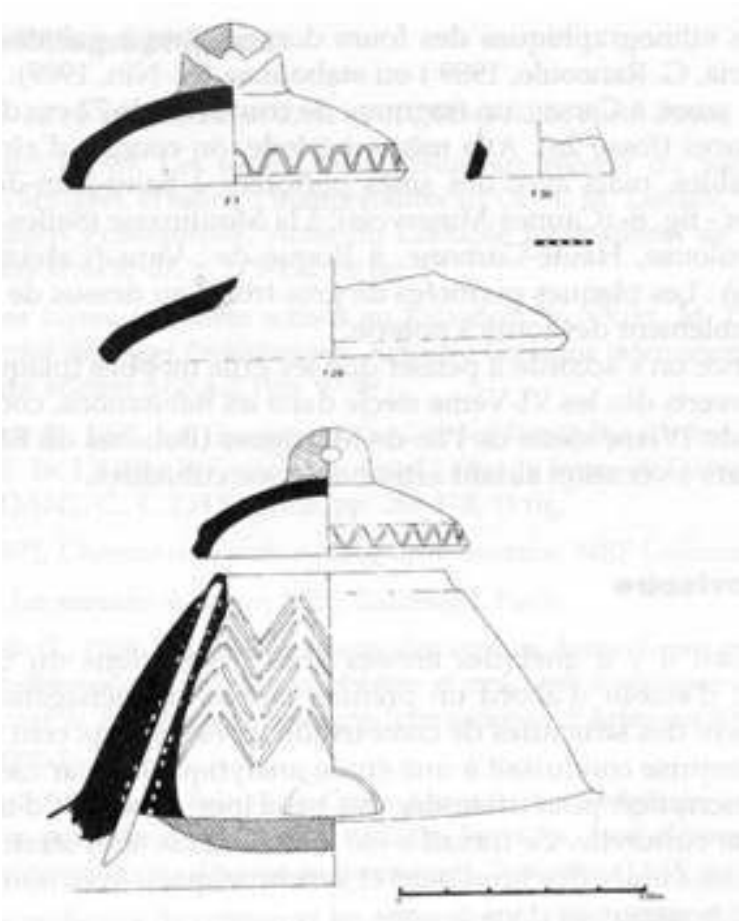

Ce four est parfois un peu rapidement rapproché des autres exemples, beaucoup plus récents, (- 450 au changement d'ère), de structures à probables cloches de cuissons (Nages, le Marduel, Pech-Maho, Martigues, Roquefavour, Rognac etc.) ou encore des cas ethnographiques des fours domestiques à galettes, de type «tannour» (D. Garcia, G. Rancoule, 1989 ) ou « tabouna » (N. Nin, 1989).

Fig. 8 : Pisé cuit de l'enceinte du Cros (Caunes-Minervois, Aude), fragment de plaque perforée cuite, probable grille ou alandier d'un four du VII-Vle siècle avant J.-C.

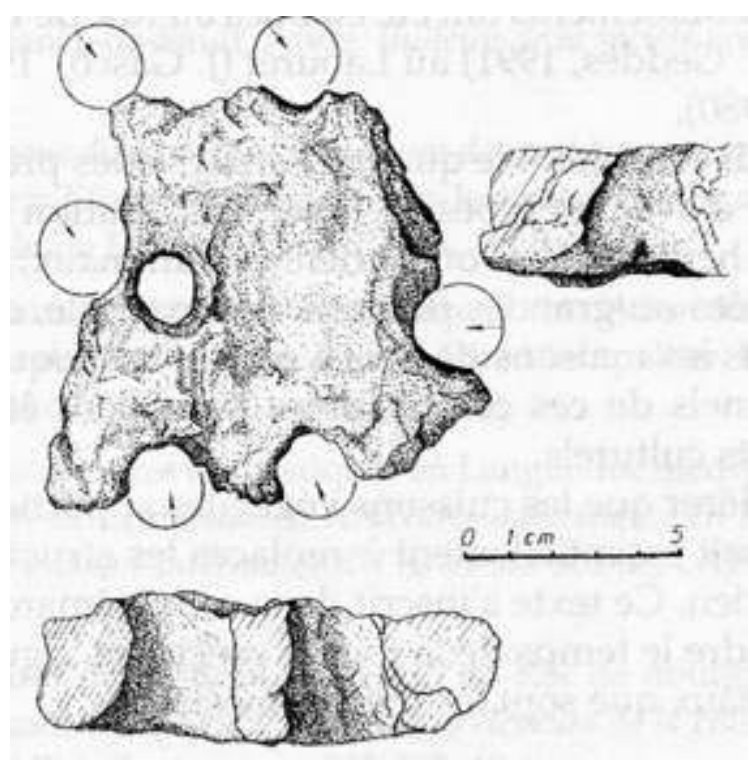


91 Signalons aussi, à Carsac, un fragment de couvercle de $72 \mathrm{~cm}$ de diamètre, cuit sur les deux faces (fosse 26). A la même période, on connaît d'autres vestiges de fours comparables, mais avec des soles perforées à Saint-Jean-de-Cas (Mailhac, Aude), au Cros Fig. 8 - (Caunes-Minervois), à la Moulinasse (Salles d'Aude, Aude), au Cluzel (Toulouse, Haute-Garonne, à Roque de Viou (Calvisson, Gard), etc. (Guilaine 1986) . Les plaques perforées de gros trous, au dessus de $3 \mathrm{~cm}$ de diamètre, sont probablement des fours à poterie.

92 En Provence on s'accorde à penser que les grils mobiles (plaques perforées en torchis) découverts dès les VI-V $\mathrm{V}^{\mathrm{e}}$ siècles dans les habitations, comme les structures élaborées du IVème siècle de l'Ile de Martigues (Bouches du Rhône) appartiennent à des fours à vocation autant artisanales que culinaires.

\section{Bilan provisoire}

93 En publiant il y a quelques années «Les Installations du quotidien ", nous avions essayé d'établir d'abord un premier corpus d'aménagements volontaires, particulièrement des structures de combustion (près de deux cent installations distinctes); l'entreprise conduisait à une étude analytique cas par cas dépassant rapidement la description pour atteindre des principes généraux d'analyse fonctionnelle et chronoculturelle. Ce travail a été limité à deux sites stratifiés qui offraient la possibilité des études diachroniques et synchroniques. Avec nous, quelques rares études ont été poursuivies dans ce sens.

94 Nous avons tenté de fixer les bases d'une méthode d'étude et celles d'un vocabulaire minimum, dans la lignée du vocabulaire d'attente d'A. Leroi-Gourhan; chaque catégorie se traduit par une forte caractérisation de la construction, de la dimension, de son fonctionnement, de son état de conservation. Tout ceci, analysé, montre l'importance de ces aménagements sur le plan de la fonction. La complémentarité des installations et les contraintes fonctionnelles de leur position dans l'organisation des établissements ont été établies au Roc de Dourgne (J. Guilaine, M. Barbaza, J. Gascó, D. Geddès, 1991) au Laouret (J. Gascó, 1996) et à l'Abri de Font-Juvénal (J. Gascó, 1980).

95 Il reste un travail considérable quant à l'analyse des produits de combustion et leur rapprochement d'avec les types de foyer. L'utilisation ou la non utilisation de types particuliers d' herbes sèches ou de fientes d'animaux, ou de bois, qui donnent chacun odeurs, fumées ou grandes flammes par exemple, explique-t-elle l'absence de certains feux dans les maisons de bois à certaines époques? Il est probable que les critères fonctionnels de ces combustibles pouvaient être aussi contrariés par toute sorte d'interdits culturels.

Il faut ici considérer que les cuissons végétales appartiennent à un petit aspect de ce travail qui visait essentiellement à replacer les structures domestiques dans leur contexte quotidien. Ce texte s'inscrit dans cette démarche et devrait inciter les archéologues à prendre le temps de la fouille précise et argumentée de ces témoins si apparemment banaux que sont les feux domestiques. 


\section{BIBLIOGRAPHIE}

ARRIG HI P., 1970, La vie quotidienne en Corse au XVIII ${ }^{e}$ siècle, Paris, Payot.

BEECHING A., GASCó J., 1989, Les foyers de la Préhistoire récente du Sud de la France, Descriptions, analyses, et essais d'interprétation. In : OLIVE, M. TABORIN, Y., dir., Nature et fonction des foyers Préhistoriques, Actes du Colloque international de Nemours 1987, Nemours, édition APRAIF, p. 275-292, 16 fig.

COUSIN F., 1989, Les foyers culinaires actuels au Rajasthan. In : OLIVE, M. TABORIN, Y., dir., Nature et fonction des foyers Préhistoriques, Actes du Colloque international de Nemours 1987, Nemours, édition APRAIF, pp. 97-99.

DAUMAS J.C., LAUDET R., 1992, Les Gandus à Saint-Ferréol-Trente-Pas (Drôme) : un habitat de pente original. In : L'habitat et l'occupation du sol à l'âge du bronze en Europe, sous la direction de Mordant, C., C.T.H.S., Paris, pp. 269-278, 11 fig.

DEFFONTAINES P., 1972, L'homme et sa maison, Géographie humaine, NRF Gallimard, Paris.

EMPERAIRE J., 1955, Les nomades de la mer, NRF, Gallimard, Paris

GARCIA D., RANCOULE G., 1989, Les aménagements des espaces domestiques protohistoriques en Languedoc-Roussillon. In : Pré-actes Habitats et structures domestiques en Méditerranée occidentale durant la Protohistoire, colloque international d'Arles-sur-Rhône, LAPMO, Aix-en-Provence, p.117-121.

GASCó J., 1979, Le néolithique de l'abri Jean Cros : déterminations végétales et techniques de consommations possibles ou probables. In : L'abri Jean Cros. Essai d'approche d'un groupe humain du Néolithique ancien dans son environnement, Toulouse, CASR, p. 371-374.

GASCó J., 1980, Les méthodes de cuisson et les structures de combustion de l'abri de Font-Juvénal Conques, Aude. In : Le groupe de Véraza et la fin des temps néolithiques dans le sud de la France et la Catalogne, Toulouse, éd. du CNRS, p. 113-114.

GASCÓ J., 1981, Les structures domestiques en Languedoc du Mésolithique à l'Age des Métaux d'après l'étude des abris de Font-Juvénal et du Roc de Dourgne dans l'Aude, Thèse pour le doctorat de $3^{\text {ème }}$ cycle, EHESS, Toulouse, 2 volumes, 611 p., 313 fig.

GASCó J., 1981, Structures domestiques du Néolithique ancien et du Mésolithique : l'abri du Roc de Dourgne Fontanès-de-Sault, Aude. Bulletin de la société préhistorique française, 78, 8, p. 227-228.

GASCó J., 1985, Les installations du quotidien, structures domestiques en Languedoc du Mésolithique à l'Age du Bronze d'après l'étude des abris de Font-Juvénal et du Roc de Dourgne dans l'Aude, Documents d'Archéologie Française, n¹, 140 p., 53 fig.

GASCÓ J., 1986, Le feu dans les sociétés néolithiques. In : Actes des séminaires 1982-1984 d’Ethnologie des régions de France, FRAYSSENGE J. Dir., Montpellier, Mission du Patrimoine - Office départemental d'action culturelle, p. 19-29.

GASCó J., 1989, Habitats et structures domestiques en Languedoc méditerranéen durant l'Age du bronze final. In : Pré-actes Habitats et structures domestiques en Méditerranée occidentale durant la Protohistoire, colloque international d'Arles-sur-Rhône, LAPMO, Aix-en-Provence, p. 36-40. 
GASCó J., 1991, Les structures domestiques de l'abri du Roc de dourgne et la diachronie. In : Dourgne. Derniers chasseurs-collecteurs et premiers éleveurs de la Haute-vallée de l'Aude, dir. GUILAINE, J., CASR CNRS, Toulouse, p. 311-323, 12 fig.

GASCÓ J. dir., 1996, Le Laouret et la Montagne d'Alaric à la fin de l'Age du Bronze, un hameau abandonné entre Floure et Monze (Aude), Archéologie en Terre d'Aude - Centre d'Anthropologie, CarcassonneToulouse, 450 p., 276 figures, 101 tableaux, photos.

GASCÓ J., 1988, L'espace domestique, reflets des activités extérieures; aménagements et structures. In : Six millénaires d'histoire de l'environnement ; étude interdisciplinaire de l'abri de Font-Juvénal (Conques-sur-Orbiel, Aude), Guilaine, J., dir.,Toulouse, Action Thématique Programmée, Histoire des milieux naturels, Centre d'Anthropologie, p.102-103, 1 fig.

GASCó J., 1989, Habitats et structures domestiques en Languedoc méditerranéen durant l'Age du Bronze final. In : Pré-actes Habitats et structures domestiques en Méditerranée occidentale durant la Protohistoire, colloque international d'Arles-sur-Rhône, Centre Nat. de la Recherche Scientifique, Aix-en-Provence, p. 36-40.

GASCó J., 1994, Vivre chez soi ; les aménagements des habitats préhistoriques in : Aude des origines, GUILAINE, J. VAQUER, J., dir. , Archéologie en Terre d'Aude, Carcassonne, p.124 ; p. 142-3 p. 162 p. 156 ; p. 173 p. 73-79, 3 fig.

GASCó J., 26-30 Septembre 1994 , L'enceinte du Cros (Caunes-Minervois, Aude) au début de l'Age du Fer, in : Les civilisations méditerranéennes, Pré-actes du XXIVe Congrès Préhistorique de France, Résumé des communications, Carcassonne, Pré-actes p.107-109.

GASCÓ J., 8-11 Mai 1997, L'enceinte du cros de Caunes-Minervois à la transition Bronze-Fer ; ouvrages architecturaux et aménagements. In : XXI ${ }^{\mathrm{e}}$ Congrès international de l'Association française pour l'étude de l'Age du Fer «Archéologie de la mort, archéologie de la tombe au premier Age du fer, Aspects de l'Age du fer dans le sud du Massif Central », Conques-Montrozier, Résumés des communications, p. 54-55, 1 ph.

GASCÓ J., COULAROU J., 1991, L'organisation des occupations à Dourgne : interprêtations, hypothèses. Dourgne. Derniers chasseurs-collecteurs et premiers éleveurs de la Haute-vallée de l'Aude, GUILAINE, J., dir., CASR CNRS, Toulouse, p. 325-342, 27 fig.

GASCó J., GILIGNY F.,LEWUILLON S., LOUIS E., LINDEMANN M., 1995, Les Structures : morphologie, catégories, chronologies, Techniques de l'archéologie en Europe, Université d'Artois, Centre National d'Enseignement à Distance, Ministère de l'Education Nationale, Arras, 90 p., 54 fig.

GASCÓ J., PERLÈs C., 1986, Le feu dans la Préhistoire. In : Actes des séminaires 1982-1984 d'Ethnologie des régions de France, FRAYSSENGE J. dir., Montpellier, Mission du Patrimoine - Office départemental d'action culturelle, p. 8-18.

GUILAINE J.,GASCÓ J.,VAQUER J., BARBAZA M.,1979, L'abri Jean Cros. Essai d'approche d'un groupe humain du Néolithique ancien dans son environnement. Centre d'Anthropologie des Sociétés Rurales, Toulouse, 462 p., 202 fig.

GUILAINE J., BARBAZA M., GASCÓ J., GEDDÈs D. et alii., 1991, Dourgne. Derniers chasseurs-collecteurs et premiers éleveurs de la Haute-vallée de l'Aude, CASR CNRS, Toulouse, 493 p., ill.

GUILAINE J., RANCOULE G.,VAQUER J., PASSELAC M., VIGNE J.D., et alii, 1986, 1 Carsac, une agglomération protohistorique en Languedoc, Centre d'Anthropologie des Sociétés Rurales, Toulouse, 271 p., 24 pl. MICHELOZZI A., 1982, L'habitation protohistorique en Languedoc oriental, A.R.A.L.O., 10, 93 p., 45 fig. 
NIN N.,1989, Les aménagements des espaces domestiques en Provence occidentale durant la Protohistoire. In : Pré-actes Habitats et structures domestiques en Méditerranée occidentale durant la Protohistoire, colloque international d'Arles-sur-Rhône, LAPMO, Aix-en-Provence, p. 122-127.

PÉTREQUIN, P. et alii, 1986, Les sites littoraux néolithiques de Clairvaux-les-lacs, I. Problématique générale, l'exemple de la station III, Maison des Sciences de l'homme, Paris, 408 p., ill.

PY M. dir., 1991, Système d'enregistrement, de gestion et d'exploitation de la documentation issue des fouilles de Lattes, Lattara 4, Lattes, 225 p., ill.

THESIGER W., 1983, Les arabes des Marais, Plon, Paris, 1959 réédition française 294 p., ill.

TILlion G, 1966, Le harem et les cousins, Ed. du Seuil, Paris, 218 p.

\section{RÉSUMÉS}

Après une présentation et le rappel de quelques principes d'études, il est proposé un premier inventaire des foyers et aménagements liés à la préparation des végétaux, particulièrement des céréales et des légumineuses pour la préhistoire récente et la protohistoire en France, essentiellement pour la zone méditerranéenne.

In this paper, domestics structures of preparation for cereal and leguminous seed (hearths and ovens) which date from the Neolithic and Protohistoric age are examined. This research has lead to a typological approach of the prehistoric domestic structures in South of France, especially in the mediterranean region.

\section{INDEX}

Mots-clés : Foyers, céréales, végétaux, néolithique, époque protohistorique, France méditerranéenne

Keywords : Hearths, cereals, plants, neolithic, Protohistoric Age, Mediterranean France

\section{AUTEUR}

\section{JEAN GASCÓ}

Centre d'Anthropologie, Unité mixte de recherche, Pôle Archéologie - Premières sociétés rurales, Université Paul Sabatier, Toulouse, France 